Southern Illinois University Carbondale

OpenSIUC

Articles

Neurology

Winter 2-17-2022

\title{
Amyloid-B(42) stimulated hippocampal lactate release is coupled to glutamate uptake
}

\author{
Erin R R. Hascup \\ Southern Illinois University School of Medicine, ehascup@siumed.edu \\ Lindsey N. Sime \\ Southern Illinois University School of Medicine \\ Mackenzie R. Peck \\ Southern Illinois University School of Medicine, mpeck76@siumed.edu \\ Kevin Hascup \\ khascup49@siumed.edu
}

Follow this and additional works at: https://opensiuc.lib.siu.edu/neurology_articles

\section{Recommended Citation}

Hascup, Erin R R., Sime, Lindsey N., Peck, Mackenzie R. and Hascup, Kevin. "Amyloid-B(42) stimulated hippocampal lactate release is coupled to glutamate uptake." Scientific Reports 12, No. 1 (Winter 2022): 2775. doi:10.1038/s41598-022-06637-2.

This Article is brought to you for free and open access by the Neurology at OpenSIUC. It has been accepted for inclusion in Articles by an authorized administrator of OpenSIUC. For more information, please contact opensiuc@lib.siu.edu. 


\title{
scientific reports
}

\section{OPEN Amyloid- $\boldsymbol{\beta}_{42}$ stimulated hippocampal lactate release is coupled to glutamate uptake}

\author{
Erin R. Hascup ${ }^{1,2}$, Lindsey N. Sime ${ }^{1}$, Mackenzie R. Peck ${ }^{1} \&$ Kevin N. Hascup ${ }^{1,2,3 凶}$
}

Since brain glucose hypometabolism is a feature of Alzheimer's disease (AD) progression, lactate utilization as an energy source may become critical to maintaining central bioenergetics. We have previously shown that soluble amyloid- $\beta(A \beta)_{42}$ stimulates glutamate release through the $\alpha 7$ nicotinic acetylcholine receptor ( $\alpha 7 n A C h R)$ and hippocampal glutamate levels are elevated in the APP/PS1 mouse model of AD. Accordingly, we hypothesized that increased glutamate clearance contributes to elevated extracellular lactate levels through activation of the astrocyte neuron lactate shuttle (ANLS). We utilized an enzyme-based microelectrode array (MEA) selective for measuring basal and phasic

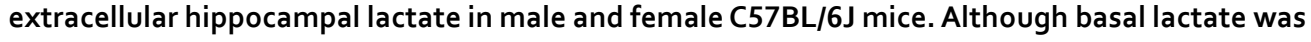
similar, transient lactate release varied across hippocampal subregions with the $\mathrm{CA} 1>\mathrm{CA} 3>$ dentate for both sexes. Local application of $A \beta_{42}$ stimulated lactate release throughout the hippocampus of male mice, but was localized to the CA1 of female mice. Coapplication with a nonselective glutamate or lactate transport inhibitor blocked these responses. Expression levels of SLC16A1, lactate dehydrogenase (LDH) A, and B were elevated in female mice which may indicate compensatory mechanisms to upregulate lactate production, transport, and utilization. Enhancement of the ANLS by $A \beta_{42}$-stimulated glutamate release during $A D$ progression may contribute to bioenergetic dysfunction in $A D$.

\author{
Abbreviations \\ a7nAChR Alpha 7 nicotinic acetylcholine receptor \\ A $\beta \quad$ Amyloid- $\beta$ \\ AA Ascorbic acid \\ AD Alzheimer's disease \\ ANLS Astrocyte-neuron lactate shuttle \\ BSA Bovine serum albumin \\ CHC 2-Cyano-3-(4-hydroxyphenyl)-2-propenoic acid \\ DA Dopamine hydrochloride \\ DG Dentate \\ DMF N,N-dimethylformamide \\ EAAT Excitatory amino acid transporters \\ FAST Fast Analytical Sensing Technology \\ LDH Lactate dehydrogenase \\ MCT Monocarboxylate transporter \\ MEA Microelectrode arrays \\ mPD 1,3 Phenylenediamine dihydrochloride \\ PBS Phosphate buffered saline \\ PU Polyurethane \\ SEM Standard error of the mean \\ TBOA DL-threo- $\beta$-benzyloxyaspartic acid \\ THF Tetrahydrofuran
}

\footnotetext{
${ }^{1}$ Department of Neurology, Dale and Deborah Smith Center for Alzheimer's Research and Treatment, Neurosciences Institute, Southern Illinois University School of Medicine, P.O. Box 19628, Springfield, IL 62794-9628, USA. ²Department of Pharmacology, Southern Illinois University School of Medicine, Springfield, IL, USA. ${ }^{3}$ Department of Medical Microbiology, Immunology and Cell Biology, Southern Illinois University School of Medicine, Springfield, IL, USA.『email: khascup49@siumed.edu
} 
Alzheimer's disease (AD) is the most prevalent form of dementia and is characterized by progressive anterograde amnesia, hippocampal atrophy, and eventual death. The majority of approved medications treat symptomatic progression, while emerging options are purported to modify disease outcome. Currently, biomarkers indicate advanced $\mathrm{AD}$ progression which limits clinical diagnosis to after memory deficits and neuronal loss. A better understanding of the neurochemical changes during the initial stages of $\mathrm{AD}$ development are needed to improve therapeutic outcome.

The hallmark pathognomonic signs of $\mathrm{AD}$ include senile plaques composed of aggregated amyloid- $\beta(\mathrm{A} \beta)_{42}$ and neurofibrillary tangles consisting of hyperphosphorylated tau. Although plaque accumulation is believed to occur earlier in disease progression ${ }^{1,2}$, soluble isoforms of $A \beta_{42}$ are neurotoxic and may precipitate $A D$ pathology $y^{3-6}$. Our laboratory has previously demonstrated that soluble $\mathrm{A} \beta_{42}$ stimulates presynaptic glutamate release through activation of the alpha 7 nicotinic acetylcholine receptor $(\alpha 7 \mathrm{nAChR})^{7}$. As AD progresses soluble $\mathrm{A} \beta_{42}$ concentrations increase leading to persistent activation of these receptors that chronically elevate extracellular glutamate levels ${ }^{8}$. This increased glutamate continuously activates postsynaptic receptors thereby dampening responses from physiological stimuli, which is hypothesized to cause the cognitive and functional decline observed in $\mathrm{AD}^{9}$. Accordingly, symptomatic effects are observed with memantine, a partial NMDA receptor antagonist, through modulation of the glutamatergic tone.

Glutamatergic signaling is terminated by uptake into $\mathrm{Na}^{+}$-dependent, high-affinity excitatory amino acid transporters (EAAT) located on glia ${ }^{10}$. The co-transport of glutamate and $\mathrm{Na}^{+}$by EAAT into astrocytes activates the $\mathrm{Na}^{+} / \mathrm{K}^{+}$ATPase to reestablish the resting membrane potential ${ }^{11}$. The ATP consumed by this pump is replenished by astrocytic glycogenolysis ${ }^{12}$ and glycolysis ${ }^{13}$ leading to lactate generation from glial glutamate uptake ${ }^{14}$. Monocarboxylate transporters (MCT) shuttle lactate out of astrocytes (MCT1 and 4) and into neurons (MCT2) where it is converted to pyruvate for use in mitochondrial oxidative phosphorylation ${ }^{15}$. This process, referred to as the astrocyte-neuron lactate shuttle (ANLS), couples neurotransmission and neuroenergetics ${ }^{11,14,16}$. The ANLS provides lactate to neurons for an additional oxidative substrate ${ }^{15}$ during increased energy demands, which is also essential for long-term memory consolidation ${ }^{12,17}$. Conversely, others have proposed the glucose sparing concept that asserts astrocytic utilization of glycogen spares their need for blood derived glucose ${ }^{18}$. This would provide neurons with enough glucose to meet energy demands during neurotransmission and memory consolidation ${ }^{19}$.

Although both neurons and astrocytes increase glycolysis after stimulation ${ }^{20,21}$, lactate is provided to neurons from astrocytes as a means to support increased energy demands ${ }^{22}$. However, brain glucose hypometabolism and vascular dysfunction are hallmark features of $\mathrm{AD}$ progression and suggests an inadequate supply of blood derived glucose to meet energy requirements. Lactate utilization as an energy source may become critical to maintaining the electrochemical gradient during $\mathrm{AD}$ progression. For example, lactate levels are elevated in the brains of amnestic mild cognitive impairment patients ${ }^{23}$, and in the cerebrospinal fluid of AD patients ${ }^{24-26}$. Cortical and hippocampal lactate levels increase with disease progression in the APP/PS1 transgenic mouse model of $\mathrm{AD}^{27}$. Based upon our previous research ${ }^{7,8}$, we hypothesized that the elevated extracellular lactate may result from increased $A \beta_{42}$ stimulated glutamate release and subsequent activation of the ANLS. To test this hypothesis, we utilized an enzyme-based microelectrode array (MEA) selective for measuring tonic and phasic extracellular lactate ${ }^{28,29}$. Changes in hippocampal extracellular lactate levels were measured in male and female $\mathrm{C} 57 \mathrm{BL} / 6 \mathrm{~J}$ mice upon local application of $\mathrm{A} \beta_{42}$ with and without EAAT and MCT inhibitors to examine the glutamate-lactate interplay as it relates to AD.

\section{Results}

Hippocampal basal and transient lactate analysis. We have previously reported spontaneous or transient release of tonic glutamate in the prefrontal cortex and hippocampus ${ }^{30,31}$. These signals were tetrodotoxin dependent, a sodium channel blocker, supporting release from synaptic signaling ${ }^{31}$. Based on these previous studies and the ANLS hypothesis, we expected to observe spontaneous tonic fluctuations in lactate signaling. Once a stable baseline was reached, MEAs were used to measure extracellular lactate levels for $10 \mathrm{~min}$ in each hippocampal subregion prior to pressure ejection recordings. Transient lactate release was observed in all hippocampal subregions and both sexes of C57BL/6J mice. Figure 1A shows representative DG, CA3, and CA1 traces from female mice. The average transient amplitude (Fig. 1B) was similar within a subregion between sexes, but was significantly different across hippocampal subregions. The largest amplitudes occurred within the CA1, followed by the CA3, and then DG of both male and female mice. Likewise, lactate transient peak area (Fig. 1C) varied across subregions with the largest in the CA1 that declined across the CA3 and DG. No differences between sexes were observed for any hippocampal subregion. The elevated transient release of lactate in the CA1 region may be in response to increased energy demands required by glutamatergic pyramidal neurons compared to granule cells types located in the DG. Despite differences in amplitude, the intertransient interval (Fig. 1D) was similar across all three subregions and sex differences were also not observed. After transient lactate analysis, basal levels were calculated without the detection of transient lactate release. Basal lactate (Fig. 1E) was increased in female C57BL/6 mice particularly within the CA3 hippocampal subregion.

Soluble $A \beta_{42}$ stimulates lactate release that is attenuated by blocking glutamate clearance. After transient and basal lactate measurements, pressure ejections studies were performed. All compounds were tested in vitro to confirm they were not electrochemically active at our recording potential prior to local application in vivo. For each compound, consistent volumes between 25 and $75 \mathrm{nl}$ were locally applied into the DG, CA3, and CA1 and no differences were observed between compounds within a brain region (Table 1). The $0.1 \mu \mathrm{M} \mathrm{A} \beta_{42}$ concentration was chosen because this elicited the maximal change in hippocampal glutamate release as shown in our previous research ${ }^{7}$. Representative lactate traces from local application in the CA1 of male C57BL/6J mice for all compounds are shown in Fig. 2A. Application of $A \beta_{42}$ elicited robust and reproduc- 

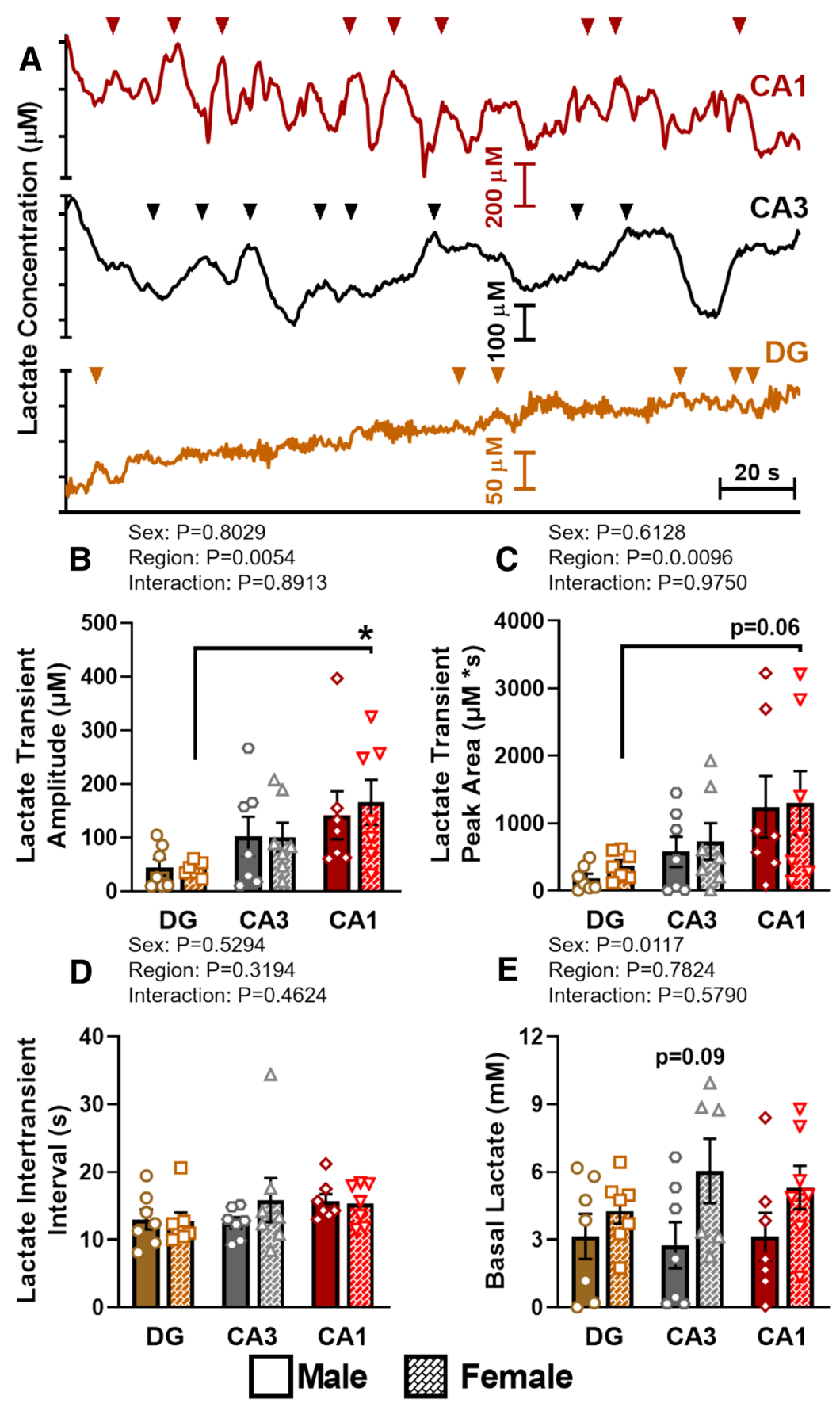

Figure 1. Transient and basal hippocampal lactate. Representative transient lactate release (A) is shown in the DG (brown), CA3 (black), and CA1 (red) of female C57BL/6 mice. Note the different scales of the ordinate $\mathrm{y}$-axis in each subregion and color-coordinated triangles identify transient release events. The average lactate transient amplitude (B), peak area (C) and intertransient interval (D) were averaged for each hippocampal region and sex. ${ }^{*} \mathrm{p}<0.05 ; \mathrm{n}=6-7$. A measurement without detectable lactate transients was used to calculate basal lactate levels $(\mathbf{E})$.

ible lactate signals. The average change in lactate amplitude from baseline for each hippocampal region is shown for male (2B) and female (2C) mice. In male mice, local application of $0.1 \mu \mathrm{M} \mathrm{A} \beta_{42}$ elicited lactate release in all hippocampal regions that was significantly higher compared to saline (vehicle control) and $0.1 \mu \mathrm{M}$ scrambled $\mathrm{A} \beta_{42}$ (negative peptide control). In female mice, lactate release from local application $0.1 \mu \mathrm{M} \mathrm{A} \beta_{42}$ was significantly higher in the CA1 compared to saline and $0.1 \mu \mathrm{M}$ scrambled $\mathrm{A} \beta_{42}$, but these effects were not observed in either the DG or CA3.

Our previous research showed soluble $A \beta_{42}$ elicited glutamate release through sodium-dependent $\alpha 7 \mathrm{nAChR}$ mechanisms supporting a role for presynaptic release. To determine if $A \beta_{42}$ mediated lactate release was coupled to glutamate uptake, $0.1 \mu \mathrm{M} \mathrm{A} \beta_{42}$ was coapplied with $100 \mu \mathrm{M}$ dl-threo- $\beta$-Benzyloxyaspartic acid (TBOA). 


\begin{tabular}{|c|c|c|c|c|c|c|c|}
\hline & \multirow[b]{2}{*}{ Hippocampal region } & \multicolumn{5}{|c|}{ Compound volume ejected (nl) } & \multirow[b]{2}{*}{ P value } \\
\hline & & Saline & Scrambled $\mathrm{A} \boldsymbol{\beta}_{42}$ & $\mathbf{A} \boldsymbol{\beta}_{42}$ & TBOA & $\mathrm{CHC}$ & \\
\hline \multirow{3}{*}{ Male C57BL/6J } & DG & $38 \pm 3$ & $39 \pm 3$ & $43 \pm 5$ & $36 \pm 2$ & $38 \pm 4$ & 0.6218 \\
\hline & CA3 & $37 \pm 2$ & $36 \pm 3$ & $42 \pm 3$ & $33 \pm 2$ & $35 \pm 3$ & 0.1270 \\
\hline & CA1 & $37 \pm 1$ & $34 \pm 3$ & $40 \pm 3$ & $37 \pm 2$ & $39 \pm 3$ & 0.5401 \\
\hline \multirow{3}{*}{ Female C57BL/6J } & DG & $38 \pm 1$ & $38 \pm 3$ & $32 \pm 2$ & $38 \pm 2$ & $40 \pm 1$ & 0.1671 \\
\hline & CA3 & $38 \pm 3$ & $38 \pm 4$ & $46 \pm 11$ & $37 \pm 3$ & $39 \pm 2$ & 0.7447 \\
\hline & CA1 & $38 \pm 2$ & $37 \pm 3$ & $43 \pm 5$ & $36 \pm 3$ & $39 \pm 1$ & \begin{tabular}{|l|l|}
0.6270 \\
\end{tabular} \\
\hline
\end{tabular}

Table 1. Average pressure ejected volumes. Volumes are shown in mean \pm SEM for application of each compound for all brain regions and sexes along with the corresponding $\mathrm{P}$ value from a one-way ANOVA. Abbreviations - $A \beta$ amyloid- $\beta$, TBOA dl-threo- $\beta$-Benzyloxyaspartic acid, $C H C$ 2-cyano-3-(4-hydroxyphenyl)2-propenoic acid.
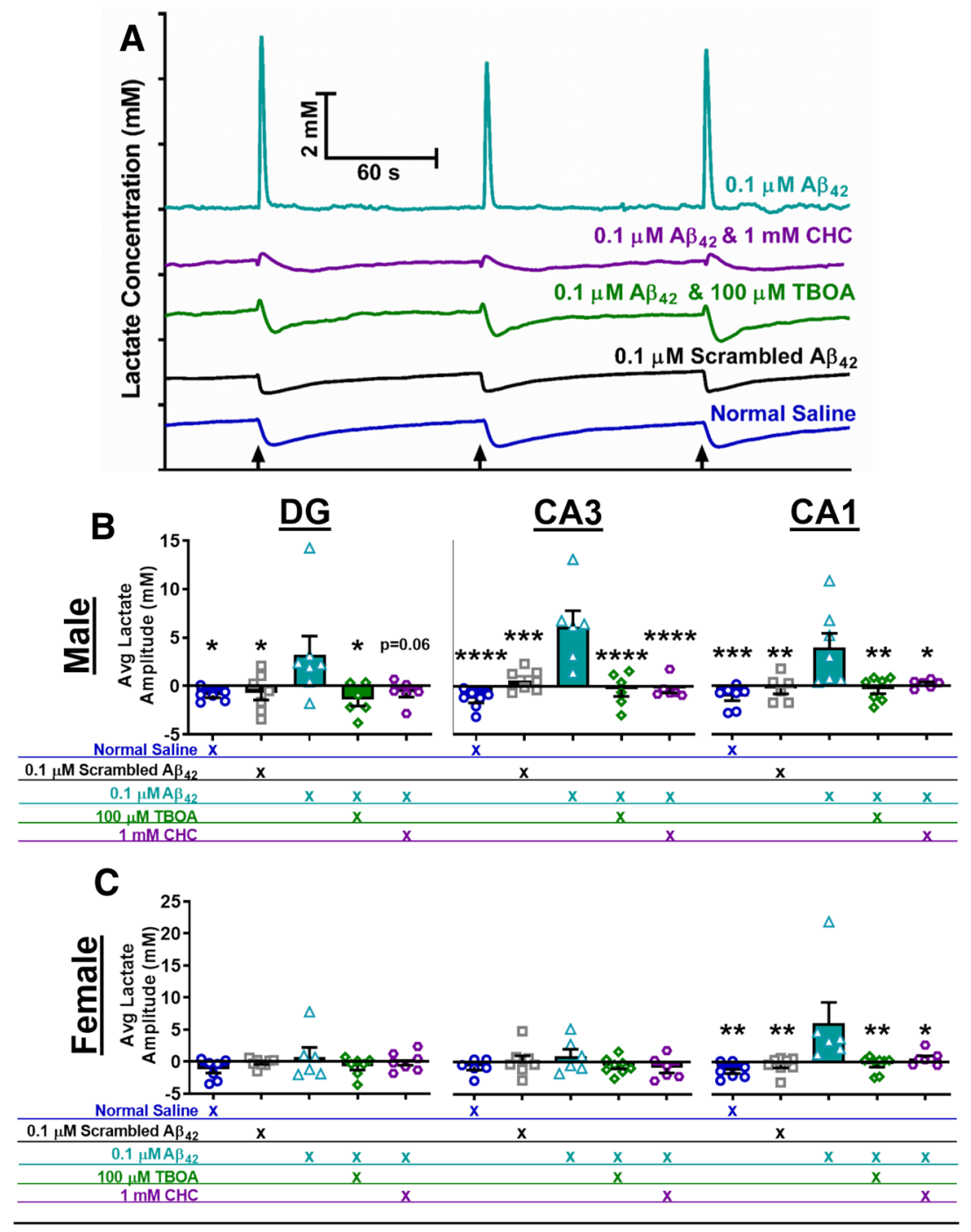

Figure 2. Efflux and inhibition of $A \beta_{42}$ stimulated lactate release. Representative traces from application of normal saline (blue), $0.1 \mu \mathrm{M}$ Scrambled $\mathrm{A} \beta_{42}$ (black), $0.1 \mu \mathrm{M} \mathrm{A} \beta_{42}$ (teal), $0.1 \mu \mathrm{M} \mathrm{A} \beta_{42}$ and $100 \mu \mathrm{M} \mathrm{TBOA}$ (green), and $0.1 \mu \mathrm{M} \mathrm{A} \beta_{42}$ and $1 \mathrm{mM} \mathrm{CHC} \mathrm{(purple)} \mathrm{are} \mathrm{shown} \mathrm{in} \mathrm{(A).} \mathrm{Traces} \mathrm{are} \mathrm{offset} \mathrm{on} \mathrm{the} \mathrm{ordinate} \mathrm{to} \mathrm{clearly}$ show lactate dynamics and black arrows indicate time point of pressure ejection. Average maximal change in lactate levels from baseline after application of each compound for each hippocampal subregion of male (B) and female (C) C57BL/6 J mice. The legend under the graph indicates the compounds that were locally (co)applied. ${ }^{*} \mathrm{p}<0.05,{ }^{* *} \mathrm{p}<0.01,{ }^{* *} \mathrm{p}<0.001,{ }^{* * *} \mathrm{p}<0.0001 ; \mathrm{n}=6-8$. 


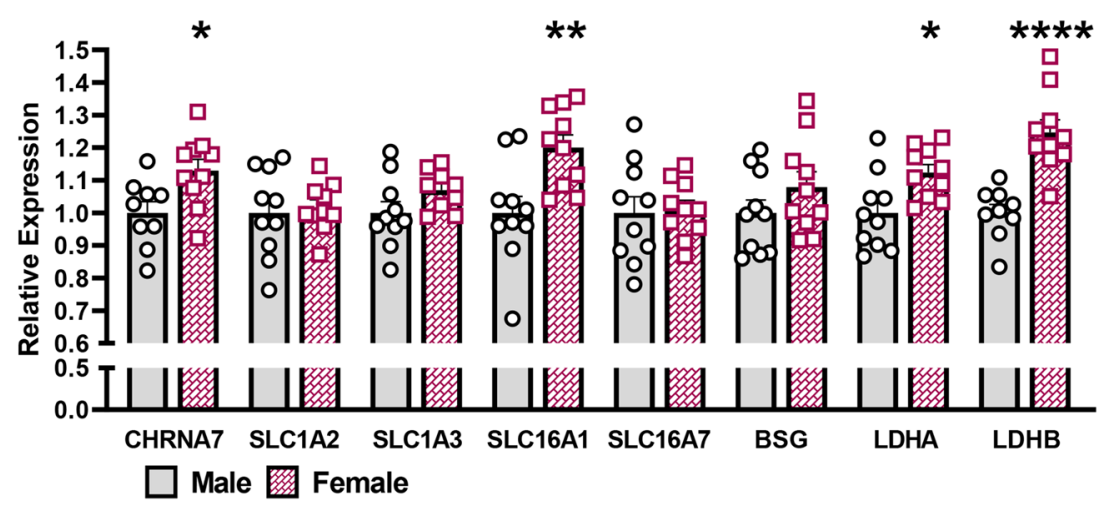

Figure 3. Hippocampal gene expression. Relative hippocampal mRNA expression levels of genes involved in the ANLS from male (gray) and female (purple) C57BL/6J mice. ${ }^{\star} \mathrm{p}<0.05,{ }^{\star *} \mathrm{p}<0.01,{ }^{\star * \star *} \mathrm{p}<0.0001 ; \mathrm{n}=9-10$.

TBOA is a competitive, non-transportable blocker of EAAT what we have previously shown to inhibit glutamate clearance from the extracellular space at these concentrations ${ }^{32}$. Coapplication of $A \beta_{42}$ with TBOA significantly attenuated the lactate release in all hippocampal subregions of male C57BL/6 J mice (Fig. 2B) and in the CA1 of female mice (Fig. 2C). Next, we coapplied $0.1 \mu \mathrm{M} \mathrm{A} \beta_{42}$ with $1 \mathrm{mM}$ 2-cyano-3-(4-hydroxyphenyl)-2-propenoic acid (CHC), a MCT inhibitor that has been shown to block glial lactate efflux at this concentration ${ }^{33}$. Coapplication of $\mathrm{A} \beta_{42}$ with $\mathrm{CHC}$ also blunted extracellular lactate levels in the hippocampus of C57BL/6J male and the CA1 region of female mice (Fig. 2B,C). These data support the increased extracellular lactate levels from local application of $A \beta_{42}$ are coupled to clearance of glutamate from the extracellular space through EAATs and efflux from MCT.

mRNA expression of glutamate and lactate transporters. To determine if sex differences in lactate release were due to varying expression levels of genes involved with the ANLS, RT-PCR was conducted on tissue from a separate, naïve, cohort of male and female mice (Fig. 3). CHRNA7 expression levels were increased in female C57BL/6J mice while glial EAATs (SLC1A2 and SLC1A3) were similar between sexes. This suggests enhanced $A \beta_{42}$-stimulated glutamate release without a change in the glutamate clearance in female mice. Next we examined expression levels involved with lactate production or utilization. Lactate dehydrogenase (LDH) is a tetramer consisting of either LDHA or LDHB subunits to form LDH-5 and LDH-1, respectively. In glial cells, homomeric LDHA catalyzes the reduction of pyruvate to lactate, while in neurons the homomeric LDHB isoform drives oxidation of lactate to pyruvate for oxidative phosphorylation ${ }^{34}$. Expression of both isoforms was increased in female mice indicating enhanced glial lactate production and neuronal utilization, respectively. While no sex differences were observed in neuronal SLC16A7 expression, we observed glial SLC16A1 expression was increased in female C57BL/6J mice. BSG is known to chaperone glial MCT1 and regulate basal and glutamate evoked lactate release through this transporter ${ }^{35}$. No BSG expression differences were observed between sexes.

\section{Discussion}

Glucose enters the brain through transporters located on astrocytic endfeet that cover the surface of blood vessels. Glucose metabolism is the predominant fuel source for the brain serving as a substrate not only for ATP production in aerobic glycolysis but also providing the carbon backbone for maintaining the neurotransmitter pools of glutamate and $\gamma$-aminobutyric acid. This ATP production is necessary to reestablish the resting membrane potential for neuronal signaling which accounts for $\sim 70 \%$ of the brain's energy expenditure with excitatory neurons accounting for $80-85 \%$ of this usage ${ }^{36}$. Lactate is a byproduct of aerobic glycolysis and during periods of increased neuronal activity, can serve as an additional energy substrate through mitochondrial oxidative phosphorylation ${ }^{11}$. In this process, neuronally released glutamate is cleared from the extracellular space through EAAT located on glia. Uptake of glutamate is coupled to glucose transport into astroglia and a subsequent increase in glycolysis. The lactate formed is shuttled from astrocytes to neurons through MCT thereby coupling glutamatergic neurotransmission with bioenergetics to support synaptic signaling.

Once thought of as a metabolic waste product, the role of lactate in brain health and disease has garnered much attention over the last two decades. During acute neurotrauma, such as with traumatic brain injury or cerebral ischemia, astrocytic release of lactate provides neuroprotection ${ }^{37}$. Lactate release is also important for memory consolidation ${ }^{12,17}$ and promotes hippocampal neurogenesis ${ }^{38}$. However, during normal aging, lactate levels increase $\mathrm{e}^{39}$ due to reduced oxidative phosphorylation from mitochondrial dysfunction. Considering aging is a risk factor for $\mathrm{AD}$, mitochondrial dysregulation and the subsequent increase in cerebral lactate is postulated to instigate disease onset ${ }^{40,41}$. For example, CSF lactate levels are increased in AD patients ${ }^{25}$. While elevated hippocampal lactate levels are observed at 12 months and correspond with cognitive deficits in the APP/PS1 amyloidogenic AD mouse mode ${ }^{27}$. At this same age in APP/PS1 mice, we reported elevated tonic levels of hippocampal glutamate ${ }^{8,42}$ that would contribute to these observed lactate levels. 
Although basal and transient lactate were similar between sexes of C57BL/6J mice, we did observe increased transient amplitude in the CA1 and CA3 compared to the DG. A couple of factors may be responsible for these differences. While histological analysis regarding the number of cells within a hippocampal subregion varies, the DG is known to have a higher density of neurons ${ }^{43}$. The increased MCT density reduces the amount of lactate present in the extracellular space. Additionally, pyramidal neurons require significantly more energy substrates to maintain synaptic integrity than granule cells resulting in larger lactate transient amplitude in the CA3 and CA1. This suggests that bioenergetic disruptions do not affect all neuronal populations equally particularly during $\mathrm{AD}$ progression.

In male mice, $A \beta_{42}$-evoked lactate release coupled to glutamate clearance was observed in all subregions, while this was only prominent in the CA1 of female mice. This suggests sexually dimorphic bioenergetics in the DG and CA3 with female mice relying on greater glucose utilization due to decreased glutamate coupled lactate release. The enhanced gene expression of glial LDHA and SLC16A1 as well as neuronal LDHB could be a compensatory mechanism to increase lactate production, transfer, and utilization to meet hippocampal bioenergetic demands. The upregulation of CHRNA7 gene expression in conjunction with soluble $A \beta_{42}$ accumulation causes excessive activation during $\mathrm{AD}$ progression. These factors coupled with the concomitant glucose hypometabolism in AD challenges the ANLS to maintain the electrochemical gradient and leaves these regions particularly vulnerable to neurodegeneration. The translational implications provides a better understanding as to the increased AD prevalence in females.

Accumulation and deposition of $A \beta_{42}$ containing senile plaques is the earliest known pathological feature associated with $\mathrm{AD}$. But, therapies targeting clearance of aggregated amyloid are only purported to modify disease progression in a subset of clinical trial participants ${ }^{44}$. This may be a consequence of how amyloid accumulation alters bioenergetics during AD progression. We have previously demonstrated soluble $A \beta_{42}$ elicits glutamate release through sodium dependent presynaptic activation of the $\alpha 7 \mathrm{nAChR} \mathrm{R}^{7}$. This mechanism contributes to elevated hippocampal tonic and phasic glutamate levels prior to onset of cognitive deficits in APP/PS1 mice ${ }^{8}$. The current study demonstrates $\mathrm{A} \beta_{42}$ also elicits lactate release coupled to glutamate uptake as a possible means to maintain energy demands for enhanced synaptic signaling based on the ANLS. Furthermore, neuronal activity is known to modulate $A \beta_{42}$ accumulation and aggregation suggestive of a vicious pathophysiological cascade ${ }^{45}$. During the initial phases of $A \beta_{42}$ accumulation, astroglia might be able to sustain the enhanced energy substrate needs. However, amyloid deposition alters the morphology and function of astroglia. These reactive astrocytes have decreased expression of EAAT 1 and 2 as well as glucose transporter $1^{46}$. This would diminish their bioenergetic capacity resulting in glucose hypometabolism and neuronal loss typified in later stages of AD progression more akin to cognitive deficits ${ }^{47}$.

The main source of oxidative substrates for neuronal energy demands is contested throughout the literature. Neurons can readily utilize both glucose and lactate as an energy substrate. But, vascular dysfunction and reduced cerebral blood flow are observed in $\mathrm{AD}^{48}$. The subsequent decrease in blood derived glucose forces neurons to rely on lactate. Additionally, synaptic glutamate release is heightened by $\mathrm{A} \beta_{42}$ activation thereby increasing ANLS as a means to meet the elevated neuronal energy demands. As soluble $\mathrm{A} \beta_{42}$ levels increase during $\mathrm{AD}$ progression, the elevated hippocampal activity ${ }^{49}$ makes larger pyramidal neurons particularly vulnerable to damage and is why they constitute a larger percentage of cell loss observed in post-mortem AD brains ${ }^{50}$. Prior to cell death, though, these damaged neurons undergo energetic inefficiency requiring additional substrates and creating competition for available resources with healthy neurons ${ }^{41}$. Eventually, the overutilization of energy substrates propagates neuronal dysfunction affecting neighboring brain regions. These changes in glutamate coupled lactate release may serve as an early biomarker for bioenergetic perturbations driving AD progression.

Limitations of the study are related to the specificity of the EAAT and MCT inhibitors. At the concentrations used, TBOA inhibits clearance of glutamate through both the glial EAAT1 and 2 and the neuronal EAAT3. Although $>90 \%$ of extracellular glutamate clearance is mediated by glial EAAT 2 in the forebrain ${ }^{10}$, neuronal uptake is a contributing factor. Likewise, $\mathrm{CHC}$ at the concentrations studied inhibits transport of lactate across either glial MCT1 or 4 and neuronal MCT2. Thus, the nonspecificity of the inhibitors used in the present study suggests a role for glutamate coupled lactate release from either neurons or glia. Future experiments with selective inhibitors or knockdown of individual MCT are needed to definitely conclude the cellular localization $\mathrm{A} \beta_{42}$ stimulated lactate release.

\section{Conclusion}

The present study provides evidence that soluble $\mathrm{A} \beta_{42}$ alters hippocampal lactate dynamics through mechanisms associated with glutamate release and clearance. Considering pathophysiological changes occur decades prior to dementia onset, the hyperexcitable neuronal networks would eventually culminate in bioenergetic dysfunction, neuronal death, and dementia progression. Hippocampal lactate dynamic differences may account for the increased incidence of dementia in females and further indicates a need for personalized patient care. Additional experiments are needed to dissect sexually dimorphic effects of lactate release in hippocampal subregions and their resulting impact on $\mathrm{AD}$ progression.

\section{Methods}

All methods were conducted in accordance with relevant guidelines and regulations.

Animals. Protocols for animal use were approved by the Institutional Animal Care and Use Committee at Southern Illinois University School of Medicine and in compliance with the ARRIVE guidelines. Male and female 3-6 month old C57BL/6J mice were obtained from Jackson Laboratory (Bar Harbor, ME; RRID:IMSR_ JAX:000664) and allowed at least 1 week to acclimate to our animal facility before electrochemical measure- 
A

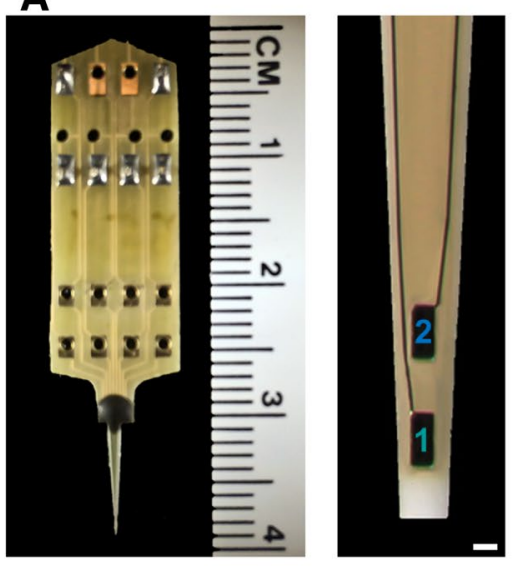

C

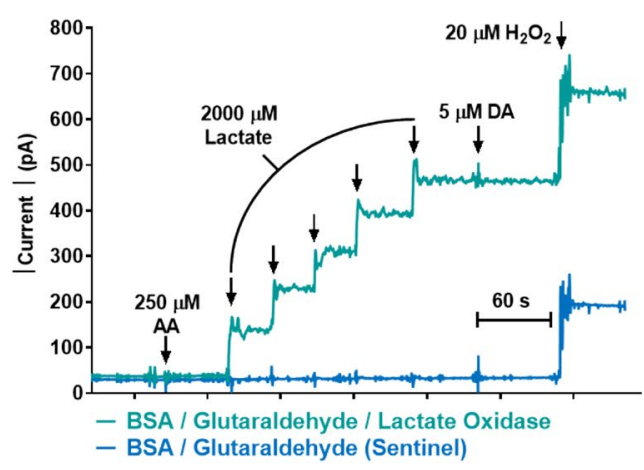

B

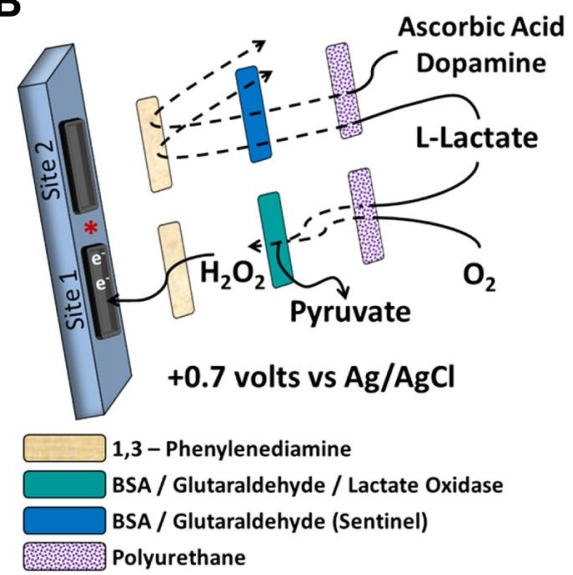

D

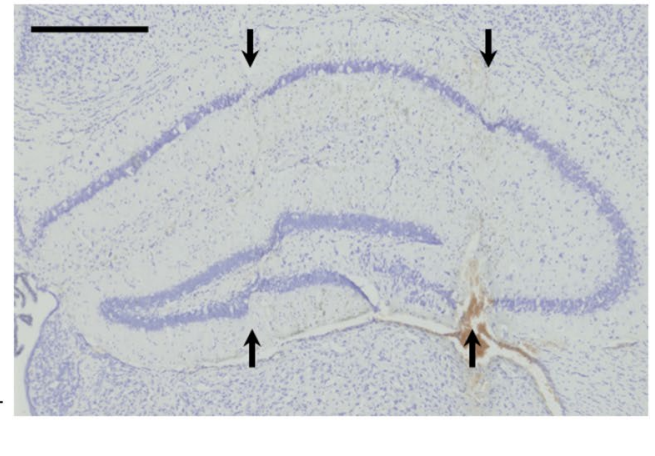

Figure 4. MEA selectivity for L-lactate hippocampal measurements. A photo of an R2 MEA with centimeter (CM) ruler for size comparison (A). A magnified view of the recording sites is shown to the right (scale bar $=50 \mu \mathrm{m}$ ). An exploded drawing of the coatings applied to the MEA for selective lactate measurements $(\mathbf{B})$. A representative calibration curve showing lactate detection only on sites coated with lactate oxidase despite both recording sites oxidizing $\mathrm{H}_{2} \mathrm{O}_{2}$ (C). A representative cresyl violet stained hippocampus (D) with probe placement tracks for the CA1 and DG (left arrows) and CA3 (right arrows). Scale bar $=500 \mu \mathrm{m}$.

ments or gene expression. Mice were group-housed according to sex and genotype on a 12:12 h light/dark schedule with food and water available ad libitum.

Chemicals. All chemicals were prepared and stored according to manufacturer recommendations unless otherwise noted. L-lactate oxidase (EC 1.1.3.2) was obtained from USBiological (Salem, MA; Cat: L3000-10) and diluted in distilled, deionized water to make a $1 \mathrm{IU} / \mu \mathrm{l}$ stock solution for storage at $4{ }^{\circ} \mathrm{C}$. Sodium phosphate monobasic monohydrate, sodium phosphate dibasic anhydrous, 1,3 phenylenediamine dihydrochloride (mPD), sodium chloride, calcium chloride dihydrate, and $\mathrm{H}_{2} \mathrm{O}_{2}$ (30\% in water) were obtained from Thermo Fisher Scientific (Waltham, MA). L-lactate sodium salt, bovine serum albumin (BSA), glutaraldehyde, dopamine hydrochloride (DA), L-ascorbic acid (AA), polyurethane (PU), tetrahydrofuran (THF), and N,N-Dimethylformamide (DMF) were obtained from Sigma-Aldrich Co. (St. Louis, MO). Human A $\beta_{1-42}$ (Cat: AS-20276) and human scrambled $\mathrm{A} \beta_{1-42}$ (Cat: AS-25383) was obtained from AnaSpec (Fremont, CA) and stored at $-80^{\circ} \mathrm{C}$ once reconstituted. CHC (Cat: 5029) and TBOA (Cat: 1223) was obtained from Tocris Bioscience (Minneapolis, MN) and stock solutions were stored at $-20^{\circ} \mathrm{C}$.

Enzyme-based MEA. MEA with platinum (Pt) recording surfaces were obtained from Quanteon LLC (Nicholasville, KY; Fig. 4A) and made selective for L-lactate recordings as previously described ${ }^{28,29}$. One microliter of lactate oxidase stock solution $(1 \mathrm{IU} / \mu \mathrm{l})$ was added to $9 \mu \mathrm{l}$ of a $1.0 \%$ BSA and $0.125 \%$ glutaraldehyde w/v solution and applied dropwise to a Pt recording surface. Lactate oxidase enzymatically degrades lactate to pyruvate and $\mathrm{H}_{2} \mathrm{O}_{2}$, the electroactive reporter molecule (Fig. $4 \mathrm{~B}$ ). The other Pt recording site (sentinel site) was coated with the BSA/glutaraldehyde solution that does not enzymatically generate $\mathrm{H}_{2} \mathrm{O}_{2}$. When a potential of $+0.7 \mathrm{~V}$ vs a $\mathrm{Ag} / \mathrm{AgCl}$ reference electrode was applied to the recording surfaces, $\mathrm{H}_{2} \mathrm{O}_{2}$ is oxidized and the current generated from the two electron transfer is amplified and digitized by the Fast Analytical Sensing Technology (FAST) 16mkIII (Quanteon LLC) electrochemistry instrument. 
PU coating. Seventy-two hours after enzyme coating, MEAs were dip coated in a $2 \%$ PU solution dissolved in a $98 \%$ THF and $2 \%$ DMF. PU limits the amount of lactate reaching the enzyme layer without blocking diffusion of $\mathrm{O}_{2}{ }^{51}$.

mPD electropolymerization. Twenty-four hours after PU coating, $10 \mathrm{mM} \mathrm{mPD}$ in $0.05 \mathrm{M}$ phosphate buffered saline (PBS) was applied to each recording surface. FAST electroplating software applied a triangular wave potential with an offset of $-0.5 \mathrm{~V}$, peak-to-peak amplitude of $0.25 \mathrm{~V}$, at a frequency of $0.05 \mathrm{~Hz}$, for $20 \mathrm{~min}$. This created a size exclusion layer that restricts the passage of AA, DA, uric acid and 3,4-dihydroxyphenylacetic acid to the Pt recording surface ${ }^{52}$.

Calibration. MEAs were placed in a stirred solution of $40.0 \mathrm{~mL}$ of $0.05 \mathrm{M}$ PBS maintained at $37^{\circ} \mathrm{C}$ using a recirculating water bath (Stryker Corp., Kalamazoo, MI) with a separate glass Ag/AgCl reference electrode (Bioanalytical Systems, Inc., West Lafayette, IN). Final beaker concentrations of $0.25 \mathrm{M} \mathrm{AA}, 2,4,6,8,10 \mathrm{M}$ Lactate, $0.005 \mathrm{M} \mathrm{DA}$, and $0.02 \mathrm{M} \mathrm{H}_{2} \mathrm{O}_{2}$ were used to determine MEA selectivity for lactate and create a standard curve for the conversion of current to lactate concentration (Fig. 4C).

Micropipette assembly and intracranial application. Glass micropipettes (World Precision Instruments, Inc., Sarasota, FL) were pulled using a vertical micropipette puller (Sutter Instrument Co., Novato, CA) with an internal diameter of 12-15 $\mu \mathrm{m}$. The micropipette tip was positioned between a pair of recording sites and mounted $100 \mu \mathrm{m}$ above the surface. All solutions applied intracranially were diluted to the appropriate concentration in physiological saline and deoxygenated under nitrogen bubbling for a minimum of $20 \mathrm{~min}$. Solutions were sterile filtered into the micropipettes. Fluids were pressure-ejected using $\mathrm{N}_{2}$ gas by a Picospritzer III (Parker-Hannafin, Cleveland, $\mathrm{OH}$ ), with pressure (5-15 psi) adjusted to consistently deliver volumes between (25-75 nl) over 1-2 s intervals. Ejection volumes were measured using a stereomicroscope Luxo Corp., Elmsford, NY) with a calibrated eye piece reticule.

Reference electrode. A Ag/AgCl reference wire was prepared by stripping the teflon coating from both ends of a silver wire (A-M Systems, Carlsberg, WA). One end was soldered to a gold-plated connector (Newark element14 Chicago, IL), while the other was placed (cathode) into a $1 \mathrm{M} \mathrm{HCl}$ bath saturated with $\mathrm{NaCl}$ that also contained a stainless steel counter wire (anode). Passing a +9 V DC to the cathode versus the anode for 15 min deposited $\mathrm{Ag} / \mathrm{Cl}$ onto the $\mathrm{Ag}$ wire.

In vivo anesthetized recordings. Mice were anesthetized using 1.5-2.0\% isoflurane (Abbott Lab, North Chicago, IL) in a calibrated vaporizer (Vaporizer Sales \& Service, Inc., Rockmart, GA) and placed in a stereotaxic frame fitted with an anesthesia mask (David Kopf Instruments, Tujunga, CA). Body temperature was maintained at $37^{\circ} \mathrm{C}$ with a water pad (Braintree Scientific Inc., Braintree, MA) connected to a recirculating water bath. A craniotomy was performed to access the dentate (DG; AP: $-2.0, \mathrm{ML}: \pm 1.0, \mathrm{DV}:-2.2 \mathrm{~mm}$ ), CA3 (AP: - 2.0, $\mathrm{ML}: \pm 2.0, \mathrm{DV}:-2.2 \mathrm{~mm}$ ) and CA1 (AP: $-2.0, \mathrm{ML}: \pm 1.0, \mathrm{DV}:-1.7 \mathrm{~mm}$ ) from Bregma ${ }^{53}$. The Ag/AgCl reference wire was positioned beneath the skull and rostral to the craniotomy thereby creating a two electrode system when the MEA was positioned in the region of interest.

Constant voltage amperometry $(4 \mathrm{~Hz}$ ) was performed with a potential of $+0.7 \mathrm{~V}$ vs the $\mathrm{Ag} / \mathrm{AgCl}$ reference electrode applied by the FAST16mkIII. MEAs reached a stable baseline for $60 \mathrm{~min}$ before basal and transient lactate analysis followed by pressure ejection studies. The FAST software saved amperometric data, time, and pressure ejection events. Calibration data, in conjunction with a MATLAB (MathWorks, Natick, MA; RRID:SCR_001622) graphic user interface program was used to calculate basal, transient, and stimulus-evoked lactate. Five evoked lactate signals in each hippocampal subfield were averaged into a representative signal for comparison. Only one compound was pressure-ejected per hemisphere for each mouse.

Cresyl violet staining. After lactate recordings, mice were euthanized with an overdose of isoflurane followed by rapid decapitation with sharp scissors. The brains were removed and placed in $4 \%$ paraformaldehyde for $24-48 \mathrm{~h}$ then stored in $30 \%$ sucrose. Twenty micron coronal sections through the hippocampus were obtained using a cryostat (Model HM525 NX, Thermo Fisher Scientific) and mounted on a glass slide. Slices were stained with cresyl violet and coverslipped. MEA placement was verified for each mouse (Fig. 4D).

RT-PCR. A separate group of 3-6 month male and female C57BL/6J mice were euthanized according to the above procedure. The brain was extracted and the hippocampus was dissected on wet ice and stored at $-80^{\circ} \mathrm{C}$ until processing. RNA was extracted from tissue by homogenization in Trizol Reagent and separated by centrifugation at $12,000 \times g$ for $15 \mathrm{~min}$ at $4{ }^{\circ} \mathrm{C}$ with chloroform. RNA was isolated by centrifugation at $12,000 \times g$ for 25 min at $4{ }^{\circ} \mathrm{C}$ in $100 \%$ isopropanol. The pellet was resuspended in RNAse free water and quantified using a NanoDrop Spectrophotometer. cDNA was synthesized using candidate primers (Integrated DNA Technologies; Table 2) and an iScript cDNA Synthesis Kit (Bio-Rad). Relative mRNA expression was analyzed by quantitative RT-PCR as previously described ${ }^{54}$ using the StepOne Real-Time PCR System (Thermo Fisher Scientific) and SYBR Green MasterMix (Bio-Rad) and using Ubiquitin-conjugating enzyme E2D2 (UBE2D2) as the internal housekeeping gene.

Statistical analysis. Prism (GraphPad Software Version 9, Inc., La Jolla, CA; RRID:SCR_002798) was used for all statistical analyses. For basal lactate and transient lactate release analysis, sexes and hippocampal brain 


\begin{tabular}{|l|l|l|l|}
\hline Gene & Name & Forward primer & Reverse primer \\
\hline BSG & Basigin & $\begin{array}{l}5^{\prime} \text {-CACCATGGCAGCCCTCTG } \\
\text { GCCC-3' }\end{array}$ & $\begin{array}{l}5^{\prime} \text {-ATAGATAAAGATGATGGTAAC } \\
\text { CAACA-3' }\end{array}$ \\
\hline CHRNA7 & $\begin{array}{l}\text { Cholinergic receptor nicotinic alpha } 7 \\
\text { subunit }\end{array}$ & $5^{\prime}$-CCTGCAAGGCGAGTTCC-3' & $\begin{array}{l}5^{\prime} \text {-CTCAGGGAGAAGTACACG } \\
\text { GTGA-3' }\end{array}$ \\
\hline LDHA & Lactate dehydrogenase A & $5^{\prime}$-ATGCACCCGCCTAAGGTTCTT-3' & $5^{\prime}$-GCCTACGAGGTGATCAAGCT-3' \\
\hline LDHB & Lactate dehydrogenase B & $5^{\prime}$-AGTCTCCCGTGCATCCTCAA-3' & $5^{\prime}$-AGGGTGTCCGCACTCTTCCT-3' \\
\hline SLC1A2 & Excitatory amino acid transporter 2 & $5^{\prime}$-CTGGTGCAAGCCTGTTTCC-3' & 5'-GCCTGTTCACCCATCTTCC-3' \\
\hline SLC1A3 & Excitatory amino acid transporter 1 & $\begin{array}{l}5^{\prime} \text {-ACCAAAAGCAACGGAGAA } \\
\text { GAG-3' }\end{array}$ & $\begin{array}{l}5^{\prime} \text {-GGCATTCCGAAACAGGTA } \\
\text { ACTC-3' }\end{array}$ \\
\hline SLC16A1 & Monocarboxylate transporter 1 & $5^{\prime}$-GGTGGGCAGTGTTAGTCGG-3' & $\begin{array}{l}5^{\prime} \text {-GATAGGACCTCCAGCATA } \\
\text { CATGA-3' }\end{array}$ \\
\hline SLC16A7 & Monocarboxylate transporter 2 & $5^{\prime}$-GGGCTGGGTCGTAGTCTGT-3' & 5'-ATCCAAGCGATCTGACTGGAG-3' $^{\prime}$ \\
\hline
\end{tabular}

Table 2. mRNA primers. Forward and reverse primers used for RT-PCR are listed for each gene.

regions were compared using a two-way ANOVA with Sidak's post hoc analysis. For A $\beta_{42}$ stimulated lactate release studies, sex and hippocampal subregions were analyzed independently with a one-way ANOVA followed by Dunnett's post hoc analysis. A two-tailed t test was used to compare hippocampal mRNA expression levels between sexes. A single Grubb's test was used to identify outliers. Bar graphs represent mean \pm standard error of the mean (SEM) and the number of animals are indicated in the figure legends. Significance was determined at $\mathrm{p}<0.05$.

\section{Data availability}

Data is available upon reasonable request.

Received: 14 October 2021; Accepted: 3 February 2022

Published online: 17 February 2022

\section{References}

1. Sperling, R. A. et al. Toward defining the preclinical stages of Alzheimer's disease: Recommendations from the National Institute on Aging-Alzheimer's Association workgroups on diagnostic guidelines for Alzheimer's disease. Alzheimers. Dement. 7, 280-292 (2011).

2. Jack, C. R. et al. Tracking pathophysiological processes in Alzheimer's disease: An updated hypothetical model of dynamic biomarkers. Lancet. Neurol. 12, 207-216 (2013).

3. Jin, M. \& Selkoe, D. J. Systematic analysis of time-dependent neural effects of soluble amyloid $\beta$ oligomers in culture and in vivo: Prevention by scyllo-inositol. Neurobiol. Dis. 82, 152-163 (2015).

4. Müller-Schiffmann, A. et al. Amyloid- $\beta$ dimers in the absence of plaque pathology impair learning and synaptic plasticity. Brain 139, 509-525 (2016).

5. Shankar, G. M. et al. Amyloid-beta protein dimers isolated directly from Alzheimer's brains impair synaptic plasticity and memory. Nat. Med. 14, 837-842 (2008).

6. Yang, T., Li, S., Xu, H., Walsh, D. M. \& Selkoe, D. J. Large soluble oligomers of amyloid $\beta$-protein from Alzheimer brain are far less neuroactive than the smaller oligomers to which they dissociate. J. Neurosci. 37, 152-163 (2017).

7. Hascup, K. N. \& Hascup, E. R. Soluble amyloid- $\beta 42$ stimulates glutamate release through activation of the $\alpha 7$ nicotinic acetylcholine receptor. J. Alzheimer's Dis. 53, 337-347 (2016).

8. Hascup, K. N., Findley, C. A., Sime, L. N. \& Hascup, E. R. Hippocampal alterations in glutamatergic signaling during amyloid progression in A $\beta$ PP/PS1 mice. Sci. Rep. 10, 14503 (2020).

9. Danysz, W. \& Parsons, C. G. Alzheimer's disease, $\beta$-amyloid, glutamate, NMDA receptors and memantine-searching for the connections. Br. J. Pharmacol. 167, 324-352 (2012).

10. Holmseth, S. et al. The concentrations and distributions of three C-terminal variants of the GLT1 (EAAT2; slc1a2) glutamate transporter protein in rat brain tissue suggest differential regulation. Neuroscience 162, 1055-1071 (2009).

11. Pellerin, L. \& Magistretti, P. J. Sweet sixteen for ANLS. J. Cereb. Blood Flow Metab. 32, 1152-1166 (2012).

12. Suzuki, A. et al. Astrocyte-neuron lactate transport is required for long-term memory formation. Cell 144, 810-823 (2011).

13. Loaiza, A., Porras, O. H. \& Barros, L. F. Glutamate triggers rapid glucose transport stimulation in astrocytes as evidenced by realtime confocal microscopy. J. Neurosci. 23, 7337-7342 (2003).

14. Pellerin, L. \& Magistretti, P. J. Glutamate uptake into astrocytes stimulates aerobic glycolysis: A mechanism coupling neuronal activity to glucose utilization. Proc. Natl. Acad. Sci. USA. 91, 10625-10629 (1994).

15. Wyss, M. T., Jolivet, R., Buck, A., Magistretti, P. J. \& Weber, B. In vivo evidence for lactate as a neuronal energy source. J. Neurosci. 31, 7477-7485 (2011).

16. Mächler, P. et al. In vivo evidence for a lactate gradient from astrocytes to neurons. Cell Metab. 23, 94-102 (2016).

17. Newman, L. A., Korol, D. L. \& Gold, P. E. Lactate produced by glycogenolysis in astrocytes regulates memory processing. PLoS ONE 6, e28427 (2011).

18. DiNuzzo, M., Mangia, S., Maraviglia, B. \& Giove, F. The role of astrocytic glycogen in supporting the energetics of neuronal activity. Neurochem. Res. 37, 2432-2438 (2012).

19. Dienel, G. A. Does shuttling of glycogen-derived lactate from astrocytes to neurons take place during neurotransmission and memory consolidation?. J. Neurosci. Res. 97, 863-882 (2019).

20. Díaz-García, C. M. et al. Neuronal stimulation triggers neuronal glycolysis and not lactate uptake. Cell Metab. 26, 361-374.e4 (2017).

21. Díaz-García, C. M. \& Yellen, G. Neurons rely on glucose rather than astrocytic lactate during stimulation. J. Neurosci. Res. 97, 883-889 (2019).

22. Barros, L. F. et al. Aerobic glycolysis in the brain: Warburg and Crabtree contra Pasteur. Neurochem. Res. 46, 15-22 (2021). 
23. Weaver, K. E. et al. Posterior cingulate lactate as a metabolic biomarker in amnestic mild cognitive impairment. Biomed Res. Int. 2015, 1-13 (2015).

24. Liguori, C. et al. CSF lactate levels, $\tau$ proteins, cognitive decline: A dynamic relationship in Alzheimer's disease. J. Neurol. Neurosurg. Psychiatry 86, 655-659 (2015).

25. Liguori, C. et al. Cerebrospinal fluid lactate levels and brain [18F]FDG PET hypometabolism within the default mode network in Alzheimer's disease. Eur. J. Nucl. Med. Mol. Imaging 43, 2040-2049 (2016).

26. Redjems-Bennani, N. et al. Abnormal substrate levels that depend upon mitochondrial function in cerebrospinal fluid from Alzheimer patients. Gerontology 44, 300-304 (1998).

27. Harris, R. A. et al. Aerobic glycolysis in the frontal cortex correlates with memory performance in wild-type mice but not the APP/ PS1 mouse model of cerebral amyloidosis. J. Neurosci. 36, 1871-1878 (2016).

28. Burmeister, J. J., Palmer, M. \& Gerhardt, G. A. l-lactate measures in brain tissue with ceramic-based multisite microelectrodes. Biosens. Bioelectron. 20, 1772-1779 (2005).

29. Lourenço, C. F., Ledo, A., Gerhardt, G. A., Laranjinha, J. \& Barbosa, R. M. Neurometabolic and electrophysiological changes during cortical spreading depolarization: Multimodal approach based on a lactate-glucose dual microbiosensor arrays. Sci. Rep. 7, 6764 (2017).

30. Hascup, K. N. et al. Resting glutamate levels and rapid glutamate transients in the prefrontal cortex of the Flinders Sensitive Line rat: A genetic rodent model of depression. Neuropsychopharmacology 36, 1769-1777 (2011).

31. Stephens, M. L. et al. Tonic glutamate in CA1 of aging rats correlates with phasic glutamate dysregulation during seizure. Epilepsia 55, 1817-1825 (2014).

32. Hascup, E. R. et al. Rapid microelectrode measurements and the origin and regulation of extracellular glutamate in rat prefrontal cortex. J. Neurochem. 115, 1608-1620 (2010).

33. Ideno, M. et al. Involvement of monocarboxylate transporter 1 (SLC16A1) in the uptake of L-lactate in human astrocytes. Life Sci. 192, 110-114 (2018).

34. Valvona, C. J., Fillmore, H. L., Nunn, P. B. \& Pilkington, G. J. The regulation and function of lactate dehydrogenase a: Therapeutic potential in brain tumor. Brain Pathol. 26, 3-17 (2016).

35. Maekawa, F., Minehira, K., Kadomatsu, K. \& Pellerin, L. Basal and stimulated lactate fluxes in primary cultures of astrocytes are differentially controlled by distinct proteins. J. Neurochem. 107, 789-798 (2008).

36. Yu, Y., Herman, P., Rothman, D. L., Agarwal, D. \& Hyder, F. Evaluating the gray and white matter energy budgets of human brain function. J. Cereb. Blood Flow Metab. 38, 1339-1353 (2018).

37. Mason, S. Lactate shuttles in neuroenergetics-homeostasis, allostasis and beyond. Front. Neurosci. 11, 43 (2017).

38. Lev-Vachnish, Y. et al. L-Lactate promotes adult hippocampal neurogenesis. Front. Neurosci. 13, 403 (2019).

39. Ross, J. M. et al. High brain lactate is a hallmark of aging and caused by a shift in the lactate dehydrogenase A/B ratio. Proc. Natl. Acad. Sci. USA. 107, 20087-20092 (2010).

40. Swerdlow, R. H. Mitochondria and mitochondrial cascades in Alzheimer's disease. J. Alzheimer's Dis. 62, 1403-1416 (2018).

41. Demetrius, L. A., Magistretti, P. J. \& Pellerin, L. Alzheimer's disease: The amyloid hypothesis and the Inverse Warburg effect. Front. Physiol. 5, 522 (2015).

42. Hascup, K. N. et al. Riluzole attenuates glutamatergic tone and cognitive decline in AßPP/PS1 mice. J. Neurochem. https://doi.org/ $10.1111 /$ jnc.15224 (2020).

43. Keller, D., Erö, C. \& Markram, H. Cell densities in the mouse brain: A systematic review. Front. Neuroanat. 12, 83 (2018).

44. Salloway, S. \& Cummings, J. Aducanumab, amyloid lowering, and slowing of Alzheimer disease. Neurology 97, 543-544 (2021).

45. Bero, A. W. et al. Neuronal activity regulates the regional vulnerability to amyloid- $\beta$ deposition. Nat. Neurosci. 14, 750-756 (2011).

46. Carter, S. F. et al. Astrocyte biomarkers in Alzheimer's disease. Trends Mol. Med. 25, 77-95 (2019).

47. Hammond, T. C. et al. $\beta$-amyloid and tau drive early Alzheimer's disease decline while glucose hypometabolism drives late decline. Commun. Biol. 3, 1-13 (2020).

48. Solis, E., Hascup, K. N. \& Hascup, E. R. Alzheimer's disease: The link between amyloid- $\beta$ and neurovascular dysfunction. J. Alzheimer's Dis. 76, 1179-1198 (2020).

49. Huijbers, W. et al. Amyloid- $\beta$ deposition in mild cognitive impairment is associated with increased hippocampal activity, atrophy and clinical progression. Brain 138, 1023-1035 (2015).

50. Mattson, M. P. \& Magnus, T. Ageing and neuronal vulnerability. Nat. Rev. Neurosci. 7, 278-294 (2006).

51. Burmeister, J. J. \& Gerhardt, G. A. Self-referencing ceramic-based multisite microelectrodes for the detection and elimination of interferences from the measurement of L-glutamate and other analytes. Anal. Chem. 73, 1037-1042 (2001).

52. Hascup, K. N. et al. Enhanced cognition and hypoglutamatergic signaling in a growth hormone receptor knockout mouse model of successful aging. J. Gerontol. A. Biol. Sci. Med. Sci. 72, 329-337 (2016).

53. Paxinos, G. \& Franklin, K. B. J. The Mouse Brain in Stereotaxic Coordinates (Gulf Professional Publishing, 2004).

54. Fang, Y. et al. Lifespan of long-lived growth hormone receptor knockout mice was not normalized by housing at $30^{\circ} \mathrm{C}$ since weaning. Aging Cell 19, 1-10 (2020).

\section{Author contributions}

E.R.H. provided funding and assisted with study design and manuscript writing. L.N.S. conducted the cresyl violet staining and MEA probe placement verification. M.P. conducted the RT-PCR analysis. K.N.H. conceived the study, conducted the experiments, analyzed the data, wrote the manuscript, and provided funding. All authors reviewed the manuscript.

\section{Funding}

This work was supported by the National Institutes of Health [NIA R01AG057767 and NIA R01AG061937], Illinois Department of Public Health [83282002F], Dale and Deborah Smith Center for Alzheimer's Research and Treatment, and the Kenneth Stark Endowment.

\section{Competing interests}

The authors declare no competing interests.

\section{Additional information}

Correspondence and requests for materials should be addressed to K.N.H.

Reprints and permissions information is available at www.nature.com/reprints.

Publisher's note Springer Nature remains neutral with regard to jurisdictional claims in published maps and institutional affiliations. 
(c) (i) Open Access This article is licensed under a Creative Commons Attribution 4.0 International cc) License, which permits use, sharing, adaptation, distribution and reproduction in any medium or format, as long as you give appropriate credit to the original author(s) and the source, provide a link to the Creative Commons licence, and indicate if changes were made. The images or other third party material in this article are included in the article's Creative Commons licence, unless indicated otherwise in a credit line to the material. If material is not included in the article's Creative Commons licence and your intended use is not permitted by statutory regulation or exceeds the permitted use, you will need to obtain permission directly from the copyright holder. To view a copy of this licence, visit http://creativecommons.org/licenses/by/4.0/.

(C) The Author(s) 2022 